\title{
Metastable photoluminescence in heavily Mg-doped GaN grown by metalorganic chemical vapor phase epitaxy
}

\author{
C. K. Shu, W. H. Lee, Y. C. Pan, H. Y. Huang, H. H. Chen, W. H. Chen, W. K. Chen, and M. C. Lee \\ Department of Electrophysics, National Chiao Tung University, Hsinchu, 300, Taiwan, R. O. C.
}

\begin{abstract}
The long-term transient spectra of heavily Mg-doped $\mathrm{GaN}$ have been investigated. As the excitation power density increased, the broad Mg-induced emission band showed blue-shift revealing characteristic of donor-acceptor pair (DAP) recombination. We also observed an unusually slow intensity decay. The characteristic time constants range from several tenths to a few hundred seconds for emission between 360 and $460 \mathrm{~nm}$. Our results are interpreted in terms of metastability due to compounded effects of differential DAP population and recombination rates and uneven acceptor distribution.
\end{abstract}

Keywords: Metastable, Mg-doped GaN, Photoluminescence

\section{INTRODUCTION}

Since the breakthrough of p-type doping in GaN-based materials, high brightness blue/green light emitting devices have been commercially available. ${ }^{1-4}$ However, it is difficult to avoid defects and impurities during material growth. To identify and even eliminate the defects are thus very needed. In recent years, some interesting phenomena related to various defects have been reported. ${ }^{5-9}$ For instance, the persistent photo-conductivity is one that can be attributed with meta-stability of energy levels within band gap. ${ }^{5,6,9}$ The photoluminescence (PL) blue shift with the increasing excitation power is another.,10 In this paper, we report an unusually slow PL intensity reduction of donor-acceptor pair (DAP) recombination in a heavily $\mathrm{Mg}$-doped GaN (GaN:Mg) film. The characteristic time constants range from several tenths to a few hundreds seconds between 360 and $460 \mathrm{~nm}$. Our results are interpreted in terms of meta-stability due to combined effects of differential DAP population and recombination rates, and non-uniform acceptor distribution that is believed to consist of broad sub-bands by overlapping shallow $\mathrm{Mg}$ acceptor levels with deep impurity band.

\section{EXPERIMENTAL}

The GaN:Mg film was grown on the (0001) sapphire substrate at $1075^{\circ} \mathrm{C}$ by metalorganic chemical vapor phase epitaxy. ${ }^{11}$ A $375 \AA$ GaN buffer layer was prepared at $520{ }^{\circ} \mathrm{C}$ before the epilayer growth. The Ga and $\mathrm{N}$ precursors were trimethylgallium (TMGa) and ammonia using the flow rates of $10.3 \mu \mathrm{mol} / \mathrm{min}$ and 0.7 standard liter per minute, respectively. For $\mathrm{Mg}$ doping, bis(cyclopentadienyl)-magnesium $\left(\mathrm{Cp}_{2} \mathrm{Mg}\right)$ diluted with hydrogen was used at a flow rate of $0.16 \mu \mathrm{mol} / \mathrm{min}$ that is equivalent to a $\mathrm{Cp}_{2} \mathrm{Mg} / \mathrm{TMGa}$ mixing ratio of 0.0154 . The $\mathrm{Mg}$ concentration was determined to be about $4.5 \times 10^{19}$ $\mathrm{cm}^{-3}$ by secondary ion mass spectrometry calibrated with a $\mathrm{Mg}$ ion-implanted standard. For the PL measurements, we utilized a He-Cd laser (Kimmon IK 5552R-F) operating ar $325 \mathrm{~nm}$ for above-band-gap excitation, and a monochromator (ARC PRO-500) and a photomultiplier tube (Hamamatsu R-955) for detection. ${ }^{12}$ The samples were held in a closed-cycle refrigerator (APD Cryogenics HC-2) for different temperature measurements between 10 and $350 \mathrm{~K}$. The incident probe power density was varied from 2.5 to $950 \mathrm{~W} / \mathrm{cm}^{2}$ by optical glass filters neglecting reflection, transmission and scattering parts. The evolution of luminescence intensity at a range from 2.84 to $3.16 \mathrm{eV}$ was also monitored by a fast data acquisition system.

\section{RESULT AND DISCUSSION}

Fig. 1 shows the PL spectra of GaN:Mg at $21 \mathrm{~K}$ with the probe power density increased from 2.5 to $925 \mathrm{~W} / \mathrm{cm}^{2}$. Only a broad violet-blue emission is observed and its intensity is seen to grow more than 1500 times (see circles in Fig. 2) and not saturated yet. This broad band shows a tail extended beyond $500 \mathrm{~nm}$ that represents the overlapping of many unresolved structures. It can be attributed to either free electron- or donor-to-deep Mg-related acceptor $\left(\mathrm{e}, \mathrm{dMg}^{0}\right.$ or $\left.\mathrm{D}^{0}, \mathrm{dMg}^{0}\right)$ transitions, ${ }^{13}$ because unintentional donor states are likely present in a heavily doped sample. The Mg-related sub-band has been 


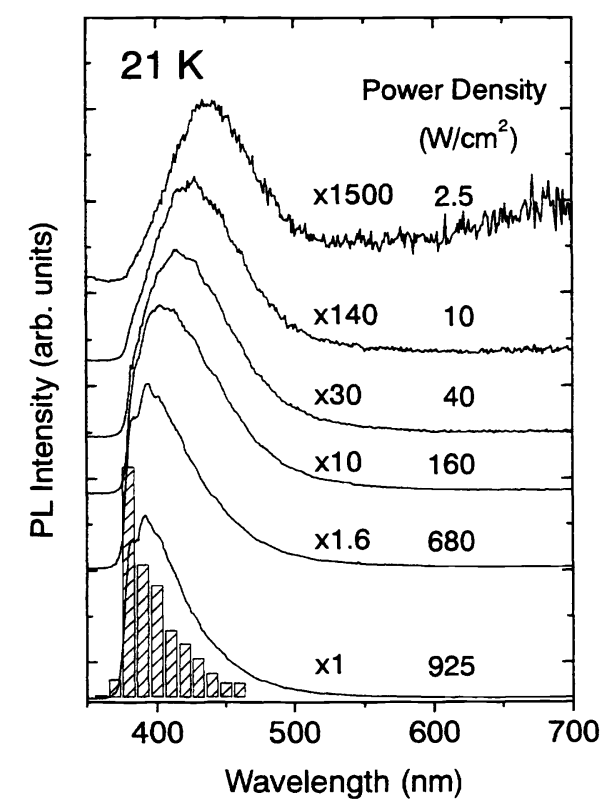

Figure 1. PL spectra of Mg-doped GaN obtained at $21 \mathrm{~K}(\lambda=325 \mathrm{~nm})$ with various probe powers.

reported as deep as $650 \mathrm{meV}$ from the valence band and yet may overlap with the shallow $\mathrm{Mg}$ acceptor $\left(\mathrm{Mg}^{0}\right)$ levels. It is due to randomly dispersed deep acceptors, ${ }^{14}$ defects and impurities (such as $\mathrm{V}_{\mathrm{Ga}}, \mathrm{V}_{\mathrm{N}}, \mathrm{Mg}_{\mathrm{Ga}}, \mathrm{O}_{\mathrm{N}}$ and $\mathrm{Mg}$-complexes). ${ }^{7,8,15-17}$ As the probe power increases, the emission profile appears less symmetric and skews towards shorter wavelength with a narrower line width. Such an excitation induced blue-shift from 2.82 to $3.16 \mathrm{eV}$ (see squares in Fig. 2) reveals the DAP characteristics, ${ }^{18}$ that is opposite to the temperature induced red-shift from band gap renormalization. ${ }^{19}$

The small peak around $392 \mathrm{~nm}$ on top of the broad band (in high power spectra) is assigned to the DAP one-phonon rep-

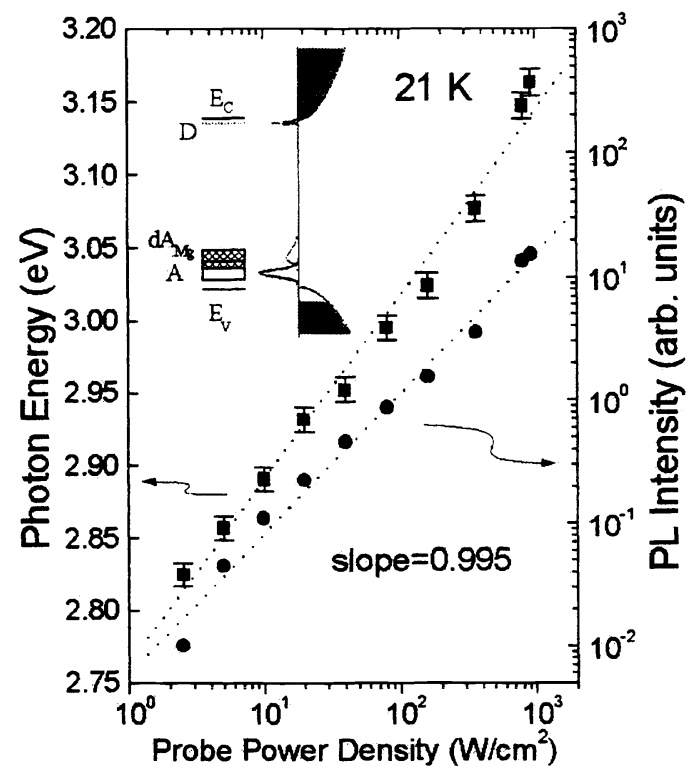

Figure 2. PL peak positions (linear) and intensities (logarithmic) vs. excitation powers in logarithmic scale. The inset shows schematically picture of the energy levels involved in the transition on the left and the corresponding distribution of states on the right. 
lica, ${ }^{10,20,21}$ since the barely resolved shoulder at $382 \mathrm{~nm}$ is commonly referred to the DAP zero-phonon line. ${ }^{22,23}$ They involve the shallow $\left(\mathrm{Mg}^{0}\right)$ levels about $220 \mathrm{meV}$ from valence band edge. To interpret the excitation induced blue shift and intensity variation, we propose in the inset of Fig. 2, a schematic energy diagram on the left with the corresponding density of states on the right. Both shallow and deep sub-bands in this diagram play important roles in the spectral distribution of the violet-blue emission. Under low probe powers, the DAPs are scarce with large separation ( $>30 \AA$, considering the Coulomb interaction, $\left.E_{D A}=E_{g}-E_{D}-E_{A}+e / 4 \pi \varepsilon r_{D A}\right)$ so that recombination is weak and in the long wavelength range as if the deep sub-band dominates the transitions. On the other hand, more localized $(<15 \AA)$ DAPs are excited under high probe powers to give off strong emission at short wavelength as if the shallow sub-band becomes dominant.

Besides, we observed the intensity reduction of the broad violet-blue emission on a time scale of several tenths to a few hundreds seconds, and found that the schematic diagram mentioned above is helpful to elucidate this behavior. The intensity evolution was monitored every $10-\mathrm{nm}$ across this band under the fixed excitation of $925 \mathrm{~W} / \mathrm{cm}^{2}$ of which only six lines are shown in Fig. 3. Through constant pumping, photo-excited electrons will either undergo free-to-bound, or relax to the shallow donor levels first and then recombine with holes in both shallow and deep sub-bands. Because of local minimum energy, ${ }^{24}$ electrons trapped in the deep sub-band usually relax slowly to the shallow sub-band and valence band. However, they may be re-excited by the continuous pumping and thus the overall recombination rate varies with time. This metastable behavior can be modeled by a simple two-intermediate-band system (using coupled rate equations) in which electron transition rate is approximately given by,

$$
I=A_{0}+A_{1}\left(1-e^{-t / \tau_{1}}\right)+A_{2} e^{-t / \tau_{2}}+A_{3} e^{-t / \tau_{3}}
$$

where $\tau_{1}$ is the rising time constant, $\tau_{2}$ and $\tau_{3}$ are the decay time constants, respectively.

As an illustration, we have simulated the upper part of the intensity evolution at $400 \mathrm{~nm}$ in Fig. 3. The circles are the experimental data and the solid line represents the least-square fit by adopting a tri-exponential function that looks extremely good. The temporal behavior can be divided into two parts, in the first 10 seconds the PL intensity increases rapidly with a time constant of $\tau_{1}=1 / k_{1}=1$ second, and afterwards it decreases slowly with two decay constants of $\tau_{2}=1 / k_{2}=39$

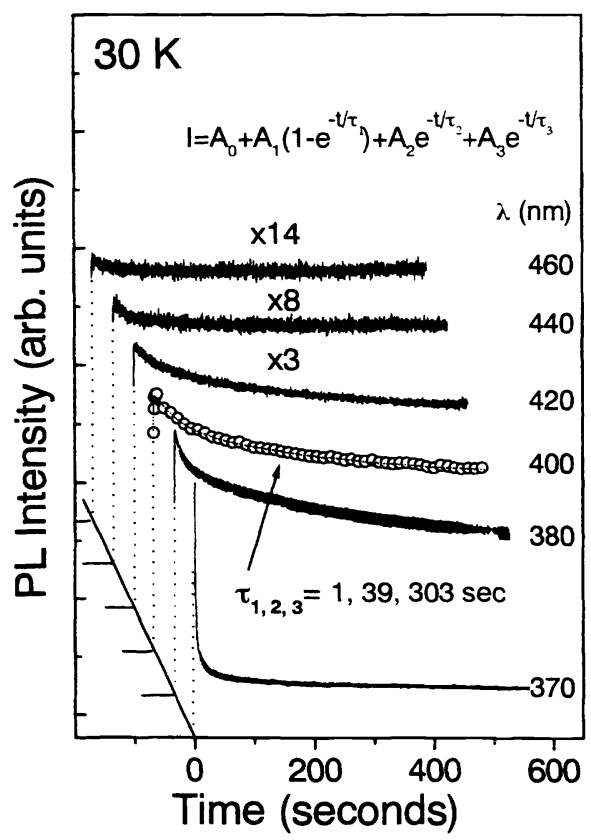

Figure 3. PL intensity reduction at different spectral positions. The solid curve at $400 \mathrm{~nm}$ is a fitting with equation

$$
I=A_{0}+A_{1}\left(1-e^{-t / \tau_{1}}\right)+A_{2} e^{-t / \tau_{2}}+A_{3} e^{-t / \tau_{3}} \text {. }
$$




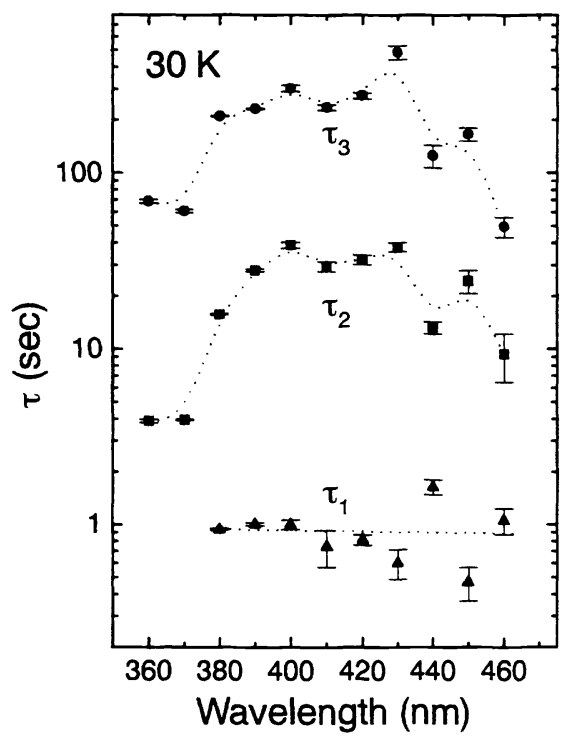

Figure 4. Three characteristic time constants for emission lines between 360 and $460 \mathrm{~nm}$.

and $\tau_{3}=1 / k_{3}=303$ seconds. The fittings for other wavelengths are also very good and their characteristic time constants are summarized in Fig. 4, in which three time constants $\left(\tau_{1}\right.$ to $\left.\tau_{3}\right)$ between 390 and $430 \mathrm{~nm}$ are in the same order range as those at $400 \mathrm{~nm}$ except that at 360,370 and $460 \mathrm{~nm}$. For emission below $380 \mathrm{~nm}$, the rising portion disappears and its decay time constants $\left(\tau_{2}\right.$ and $\left.\tau_{3}\right)$ are almost one order of magnitude shorter than those above $390 \mathrm{~nm}$. Thus, the recombination mechanisms for shallow sub-band are different from deep one. From Eq. (1) the products $A_{1} \tau_{1}, A_{2} \tau_{2}$ and $A_{3} \tau_{3}$ represent the time integrated intensities. Fig. 5 shows the emission energy dependence of the three components $\left(A_{1} \tau_{1}\right.$, $\left.A_{2} \tau_{2}, A_{3} \tau_{3}\right)$ extracted from a series of decay curves. The profile of the rising component $A_{1} \tau_{1}$ looks like an exponential function versus wavelength. However, we cannot detect the $A_{1} \tau_{1}$ at the 360 and $370 \mathrm{~nm}$, because the short decay time $\tau_{2}$ ( $4 \mathrm{sec}$ ) overshadows the rising time $\tau_{1}$ at the same wavelength. The $A_{2} \tau_{2}$ profile resembles closely to the $A_{3} \tau_{3}$ profile and indicates that the decay components $\tau_{2}$ and $\tau_{3}$ represent the similar recombination deep traps. The asymmetric profile of $A_{2} \tau_{2}$ or $A_{3} \tau_{3}$ also reflects in the highest excitation spectrum of Fig. 1 about $390 \mathrm{~nm}$.

On the other hand, different time constants would cause serious distortion in the spectral line shape, especially when one uses a scanning spectrometer combined with a single channel detector (photo-multiplier tube, PMT). Since it may take 5 to 15 minutes to acquire a typical PL spectrum, the final spectral distribution will be dominated by the emissions having long time constants. We show such an effect as the crosshatched area in Fig. 1, where the histograms of the integrated PL intensity over a 10-second period in the interval from 50 to 150 seconds are drawn from the emissions displayed in Fig. 3. Evidently, the main portion of luminescence is from emissions having long time constants. In order to correct the aforementioned spectral distortion, it is preferable to use a multi-channel detector that is able to record signals simultaneously over a wide spectral range. Besides, a proper integration time is very crucial for a true spectrum without significant distortion. If the multiple exposure method were used, one could even obtain the temporal evolution of the entire spectral distortion.

Regarding the time constant variation, there are at least two factors involved in the DAP recombination rate. First, the subband distribution is not necessarily flat, but likely bulging at roughly the sub-band minimum (see the inset of Fig. 2). This means that more DAPs available for the transition to occur at higher energy than lower energy. Under the same pumping power, it will take longer time for the intensity to reduce that is actually reflected by the longer time constants between 430 


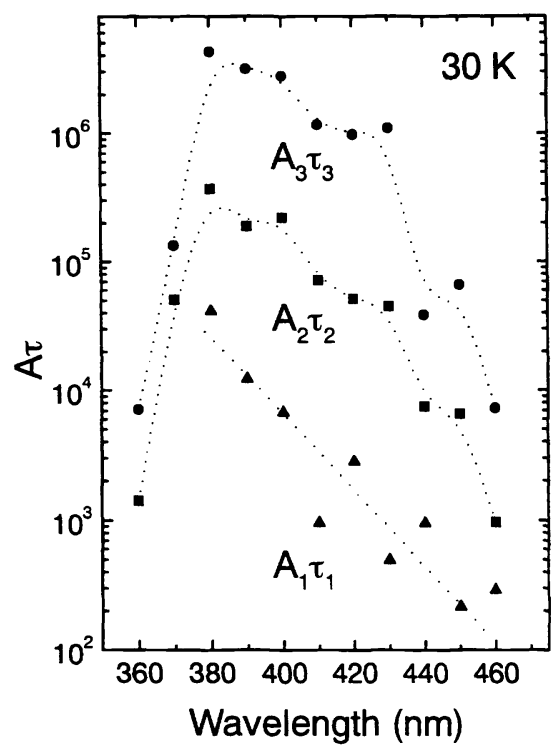

Figure 5. The time integrated intensities $A_{1} \tau_{1}, A_{2} \tau_{2}$ and $A_{3} \tau_{3}$ for emission lines between 360 and $460 \mathrm{~nm}$.

and $390 \mathrm{~nm}$. Secondly, the recombination rate for the high energy DAPs is greater than that for the low energy pairs because of smaller separation. Greater transition rate causes faster intensity reduction and hence shorter time constant as shown by $\tau_{2}=16$ seconds at $380 \mathrm{~nm}$.

The long decay times indicate that electrons or holes are likely trapped into metastable states. We believe that the slow PL intensity reduction at $30 \mathrm{~K}$ is due to finite unoccupied acceptors in the sub-band. Once the pumping starts DAP recombination, the population of unoccupied acceptors begins to deplete and hence reduces the number of DAPs for transitions. This gradually quenches the recombination process and diminishes the luminescence intensity. At room temperature, the intensity reduction is insignificant, instead it is observed to rise at the commencement of pumping. As the time goes by, it rises further and eventually reaches the plateau of steady state. We have measured a very long characteristic time of 650 seconds at $450 \mathrm{~nm}$ and noticed that the emission color changes gradually from violet to blue (observable with naked eye). It can be attributed to thermal quenching of unoccupied acceptors in the sub-bands which limits the DAP recombination rate and forces an equilibrium state of pumping and de-excitation to prevail.

\section{SUMMARY}

In conclusion, we have observed the power dependent blue-shift and intensity variation in Mg-doped GaN film that suggest DAP transition characteristics. Luminescence intensity decay was studied at $30 \mathrm{~K}$ with the time constants varying from a few to several hundreds seconds for emission between 360 to $460 \mathrm{~nm}$. The transition mechanism and influencing factors involved in the violet-blue emission were discussed in terms of metastability due to compounded effects of uneven acceptor distribution and differential DAP population and recombination rate.

\section{ACKNOWLEDGMENTS}

The authors wish to acknowledge the support by the National Science Council of Republic of China under contract numbers NSC88-2112-M009-021, 022 and 031.

\section{REFERENCE}


1. Proceedings of The Second International Conference on Nitride Semiconductors, ICNS'97, Tokushima, Japan, Oct. 2731, 1997.

2. M. Kneissl, D. P. Bour, N. M. Johnson, L. T. Romano, B. S. Krusor, R. Donaldson, J. Walker, and C. Dunnrowicz, "Characterization of AlGaInN diode lasers with mirrors from chemically assisted ion beam etching," Appl. Phys. Lett. 72, 1539, 1998.

3. Proceedings of The Second International Symposium on Blue Laser and Light Emitting Diodes, 2nd ISBLLED, Kisarazu, Chiba, Japan, p 371, Sep. 29 - Oct. 2, 1998.

4. S. Nakamura, M. Senoh, N. Iwasa, S. Nagahama, T. Yamada, and T. Mukai, Jpn. J. Appl. Phys. Part 2 34, L1332, 1995.

5. M. T. Hirsch, J. A. Wolk, W. Walukiewicz, and E. E. Haller, "Persistent photoconductivity in n-type GaN," Appl. Phys. Lett. 71, 1098, 1997.

6. C. Johnson, J. Y. Lin, H. X. Jiang, M. Asif Khan, and C. J. Sun, "Metastability and persistent photoconductivity in Mgdoped p-type GaN,” Appl. Phys. Lett. 68, 1808, 1996.

7. U. Kaufmann, M. Kunzer, M. Maier, H. Obloh, A. Ramakrishnan, B. Santic, and P. Schlotter, "Nature of the $2.8 \mathrm{eV}$ photoluminescence band in Mg doped GaN," Appl. Phys. Lett. 72, 1326, 1998.

8. E. Oh, H. Park, and Y. Park, "Excitation density dependence of photoluminescence in GaN:Mg," Appl. Phys. Lett. 72, 70, 1998.

9. J. Z. Li, J. Y. Lin, H. X. Jiang, A. Salvador, A. Botchkarev, and H. Morkoc, "Nature of Mg impurities in GaN," Appl. Phys. Lett. 69, 1474, 1996.

10. L. Eckey, U. von Gfug, J. Holst, A. Hoffmann, A. Kaschner, H. Siegle, C. Thomsen, B. Schineller, K. Heime, M. Heuken, O. Schön, and Beccard, "Photoluminescence and Raman study of compensation effects in Mg-doped GaN epilayers,” J. Appl. Phys. 84, 5828, 1998.

11. H. C. Lin, C. K. Shu, J. Ou, Y. C. Pan, W. K. Chen, and M. C. Lee, "Growth temperature effects on In $\mathrm{Ga}_{1-x} \mathrm{~N}$ films studied by X-ray and photoluminesce,"J. Crys. Growth, 189-190, 57, 1998.

12. C. K. Shu, J. Ou, H. C. Lin, W. K. Chen, and M. C. Lee, "Isoelectronic In-doping effect in GaN films grown by metalorganic chemical vapor deposition," Appl. Phys. Lett. 73, 641, 1998.

13. D. J. As, T. Simonsmeier, B. Schöttker, T. Frey, D. Schikora, W. Kriegseis, W. Burkhardt, and B. K. Meyer, "Incorporation and optical properties of magnesium in cubic GaN epilayers grown by molecular beam epitaxy," Appl. Phys. Lett. 73, 1835, 1998.

14. H. C. Casey, Jr., J. Muth, S. Krishnankutty, and J. M. Zavada, "Dominance of tunneling current and band filling in InGaN/AlGaN double heterostructure blue light-emitting diodes," Appl. Phys. Lett. 68, 2867, 1996.

15. J. Neugebauer and C. G. Van de Walle, "Atomic geometry and electronic structure of native defects in GaN," Phys. Rev. B 50, 8067, 1994.

16. M. Smith, G. D. Chen, J. Y. Lin, H. X. Jiang, A. Salvador, B. N. Sverdlov, A. Botchkarev, H. Morkoc, and B. Goldenberg, "Mechanisms of band-edge emission in Mg-doped p-type GaN," Appl. Phys. Lett. 68, 1883, 1996.

17. P. Boguslawski, E. L. Briggs, and J. Bernholc, "Native defects in gallium nitride," Phys. Rev. B 51, 8067, 1995.

18. P. Y. Yu, M. Cardona, Fundamentals of Semiconductors - Physics and Materials, Springer-Verlag Berlin Heidelberg, New York, p. 344-349, 1996.

19. J. M. Myoung, K. H. Shim, C. Kim, O. Gluschenkov, K. Kim, S. Kim, D. A. Tumbull, and S. G. Bishop, “Optical characteristics of p-type GaN films grown by plasma-assisted molecular beam epitaxy,” Appl. Phys. Lett. 69, $2722,1996$.

20. G. Popovici, G. Y. Xu, A. Botchkarev, W. Kim, H. Tang, A. Salvador, H. Morkoc, R. Strange, and J. O. White, "Raman scattering and photoluminescence of $\mathrm{Mg}$-doped $\mathrm{GaN}$ films grown by molecular beam epitaxy," J. Appl. Phys. 82, 4020, 1997.

21. S. Fischer, C. Wetzel, E. E. Haller, and B. K. Meyer, "On p-type doping in GaN-acceptor binding energies," Appl. Phys. Lett. 67, 1298, 1995.

22. M. Leroux, B. Beaumont, N. Grandjean, P. Lorenzini, S. Haffouz, P. Vennéguès, J. Massies, P. Gibart, "Luminescence and reflectivity studies of undoped, n- and p-doped GaN on (0001) sapphire," Mater. Sci. Eng. B50, 97, 1997.

23. G. P. Yablonskii, A. L. Gurskii, E. V. Lutsenko, I. P. Marko, B. Schineller, A. Guttzeit, O. Schön, M. Heuken, K. Heime, R. Beccard, D. Schmitz, and H. Juergensen, "Optical properties and recombination mechanisms in GaN and GaN:Mg grown by metalorganic vapor phase epitaxy," J. Elec. Mater. 27, 222, 1998.

24. P. Bäume, J. Gutowski, D. Wiesmann, R. Heitz, A. Hoffmann, E. Kurtz, D. Hommel, and G. Landwehr, "Intensitydependent energy and line shape variation of donor-acceptor-pair bands in $\mathrm{ZnSe}: \mathrm{N}$ at different compensation levels," Appl. Phys. Lett. 67, 1914, 1995.

25. A. A. Istratov, and O. F. Vyvenko, "Exponential analusis in physical phenomena," Rev. Sci. Inst. 70, $1233,1999$. 